\title{
Differential Expression of cdc25 Cell-Cycle-Activating Phosphatases in Human Colorectal Carcinoma
}

\author{
Silvia Hernández, Xavier Bessa, Sílvia Beà, Lluis Hernández, Alfons Nadal, \\ Carme Mallofré, Jaume Muntane, Antoni Castells, Pedro L. Fernández, \\ Antonio Cardesa, and Elias Campo
}

Laboratory of Pathology (SH, SB, LH, CM, JM, PLF, ACar, EC) and Department of Gastroenterology (XB, ACas), Hospital Clínic, and Hospital Casa de Maternitat (AN), Institut d'Investigacions Biomediques August Pi i Sunyer (IDIBAPS), University of Barcelona, Barcelona, Spain

SUMMARY: $c d c 25$ is a family of cell-cycle phosphatases that activate the cyclin-dependent kinases. cdc25A and $B$, but not $C$, have oncogenic potential in vitro. In this study, we analyzed the possible implication of cdc25 genes in the progression of colorectal tumors. RNA and DNA were extracted from 34 paired tumor and normal colorectal tissues and examined by Northern blot, RT-PCR, and Southern blot, respectively. Protein expression was analyzed by Western blot in a subset of normal and tumor samples. The expression levels were correlated with the clinicopathologic characteristics and survival of the patients. cdc25B mRNA was overexpressed in 19 carcinomas (56\%). A significant correlation was observed between high cdc25B mRNA levels and the relapse-free, overall, and cancer-related survival of the patients. The cdc25B2 splicing variant was detected in 27 carcinomas $(79 \%)$ but only in 9 normal samples (26\%) and was associated with the grade of the differentiation of the tumors. cdc25A mRNA was overexpressed in four tumors (12\%) and cdc25C1 mRNA was overexpressed in nine tumors (26\%). A new cdc25C2 splicing variant lacking exon 4 and 5 was identified in all of the tumors and in $56 \%$ of the normal samples. No amplifications or gene rearrangements of these genes were detected. In conclusion, these findings indicate that cdc25 isoforms and splicing variants are differentially regulated in colorectal carcinomas and may participate in the development of these tumors. Additionally, the correlation between cdc25B mRNA levels and the survival of the patients also suggest that the cdc25B isoform may be involved in the progression of the disease. (Lab Invest 2001, 81:465-473).

D eregulation of cell-cycle control is believed to be an essential event in the malignant transformation of cells. Alterations in cell-cycle regulatory genes are frequently found in cancer models (Hunter and Pines, 1994; Sherr, 1996). Cyclin-dependent kinases (CDK) and their regulatory subunit cyclins are essential proteins in cell-cycle progression. The different CDK/ Cyclin complexes are activated and inactivated sequentially, allowing the cell to progress through the different phases of the cell cycle (Sherr, 1996). CDK are positively regulated by association with specific cyclins and negatively regulated by different CDK inhibitors belonging to the CIP/KIP (p21 Waf1, p27 Kip1, p57 ${ }^{\text {Kip2 }}$ ) and INK4 (p16 ${ }^{\text {INK4a }}, p 15^{\text {INK4b }}, p 18^{\text {INK4c }}, p 19^{\text {INK4d }}$ families (Sherr and Roberts, 1995). CDK activity is also regulated by a series of phosphorylations of different residues. In particular, phosphorylation by wee- 1 kinase of the Thr-14 and Tyr-15 residues in the ATP-binding site inhibits CDK activity (Igarashi et al, 1991; Norbury et al,

\section{Received October 26, 2000.}

This work was supported by Comisión Interministerial de Ciencia y Tecnologia (CYCIT) Grant SAF 99/20, Asociación Española Contra el Cáncer (AECC), Fundació Rius $i$ Virgili, and Generalitat de Catalunya CIRIT 98SGR21.

Address reprint requests to: Dr. Elias Campo, Laboratory of Pathology, Hospital Clinic, Villarroel 170, 08036-Barcelona, Spain. E-mail: campo@medicina.ub.es
1991), whereas dephosphorylation of these residues by cdc25 phosphatases activates CDK, allowing the cell to progress in the cell cycle.

cdc25 is a family of human CDK-activating phosphatases composed of three different members, cdc25A, cdc25B, and cdc25C, which seem to act in different points of the cell cycle (Galaktionov and Beach, 1991; Jinno et al, 1994; Nagata et al, 1991; Sadhu et al, 1990). cdc25A is expressed in late G1 phase and is essential for G1/S transition by activating the cyclin E/CDK2 complex (Hoffmann et al, 1994; Jinno et al, 1994), whereas cdc25B and C mainly regulate $\mathrm{G} 2$ and G2/M transition (Hoffmann et al, 1993; Izumi and Maller, 1993; Lammer et al, 1998; Strausfeld et al, 1994). In addition to these cdc25 phosphatases, alternate splicing of a single pre-mRNA may produce at least five cdc25B variants, which seem to be differentially expressed in several cell types and may have different functional efficiencies in regulating mitosis (Baldin et al, 1997; Forrest et al, 1999). However, the possible expression of these splicing variants and their potential role in the pathogenesis of human tumors is not well known.

Studies have indicated a potential oncogenic effect of cdc25 phosphatases in experimental models and human tumors. Particularly, cdc25A and $B$, but not $C$, cooperate with activated Ha-ras or loss of $R b 1$ in the transformation of murine fibroblasts (Galaktionov et al, 
1995). They may also function as downstream elements of c-myc or E1A oncogenic pathways (Galaktionov et al, 1996; Spitkovsky et al, 1996). Deregulated overexpression of cdc25B in transgenic mice induces mammary gland hyperplasia and increases susceptibility to induction of mammary tumors (Ma et al, 1999; Yao et al, 1999). Furthermore, cdc25A and B overexpressions have been observed in different cancer cell lines and human tumors, suggesting that these phosphatases are implicated in human neoplasms (Galaktionov et al, 1995; Gasparotto et al, 1997; Hernández et al, 1998, 2000; Kudo et al, 1997; Takemasa et al, 2000; Wu et al, 1998).

Malignant transformation of colorectal mucosa is associated with a progressive increase in cell proliferation from normal epithelium to adenoma and carcinoma (Risio et al, 1988). Several studies have demonstrated alterations in different cell-cycle regulatory genes in these tumors, including overexpression of the activators cyclin D1 and cdc2/CyclinA and deregulation of inhibitors such as p21 Waf1, p2 $7^{\mathrm{Kip} 1}$, and p16 ${ }^{\text {INK4a }}$ (el-Deiry et al, 1995; Esteller et al, 2000; Girlando et al, 1999; Herman et al, 1995; Sgambato et al, 1999; Viale et al, 1999; Yamamoto et al, 1998). However, the possible role of the different cell-cycleactivating cdc25 phosphatases in the development of colorectal tumors has been less examined (Takemasa et al, 2000). In the present study, we analyzed the mRNA and protein expression and the gene structure of the three cdc25 isoforms, A, B, and C, including their splicing variants, in a series of cancer and normal colon tissues. We also evaluated the relationship of these alterations to the pathologic characteristics of the tumors and the evolution of the patients.

\section{Results}

\section{Differential Expression of cdc25 Isoforms}

cdc25A. cdc25A expression was analyzed by Northern blot and RT-PCR in 34 paired normal and cancer colon tissues. In the Northern blot analysis, the control cell line Molt-4 showed a relatively strong mRNA signal. However, no detectable cdc25A expression was seen in any of the normal or tumor colorectal samples (Fig. 1). We used a semiquantitative RT-PCR technique to determine whether cdc25A was expressed at lower levels in these tissue samples. A specific cdc25A signal was detected in all cases with similar expression levels in normal and tumor samples. Four tumors (12\%) in Stage II had mRNA overexpression (tumor to normal ratio $>3$-fold) compared with the corresponding normal tissues (Fig. 2).

cdc25A protein expression was examined in 15 tumors and their corresponding normal mucosa by Western blot (Fig. 3). Contrary to mRNA analysis, protein overexpression (tumor to normal ratio $>$ 3-fold) was detected in a higher number of carcinomas (8 of 15, 53\%). The correlation between protein and mRNA expression was concordant in nine tumors (60\%), with two tumors overexpressing both cdc25A mRNA and protein and seven tumors showing similar

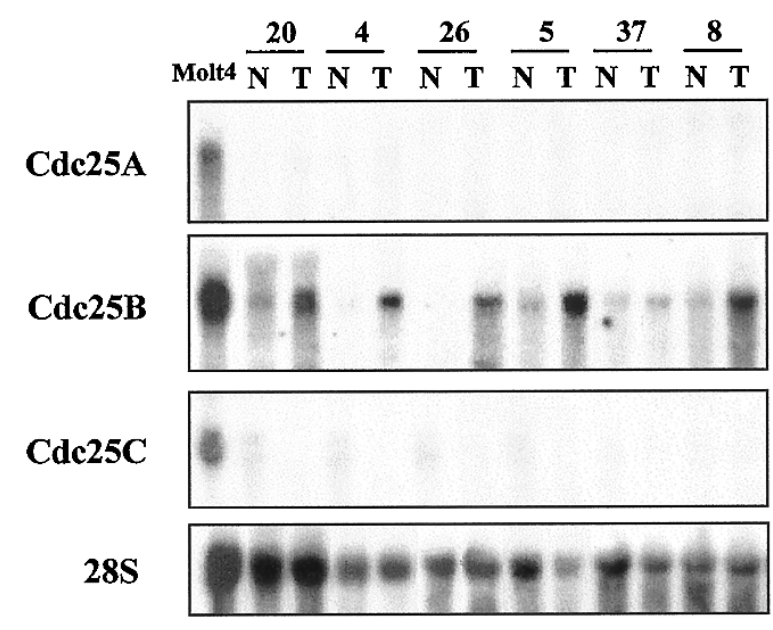

Figure 1.

Northern blot analysis of cdc25A, B, and C in a series of tumor (T) and normal (N) human colon samples. cdc25B is overexpressed in colon tumors. No cdc25A and cdc25C expression was detected by Northern blot.

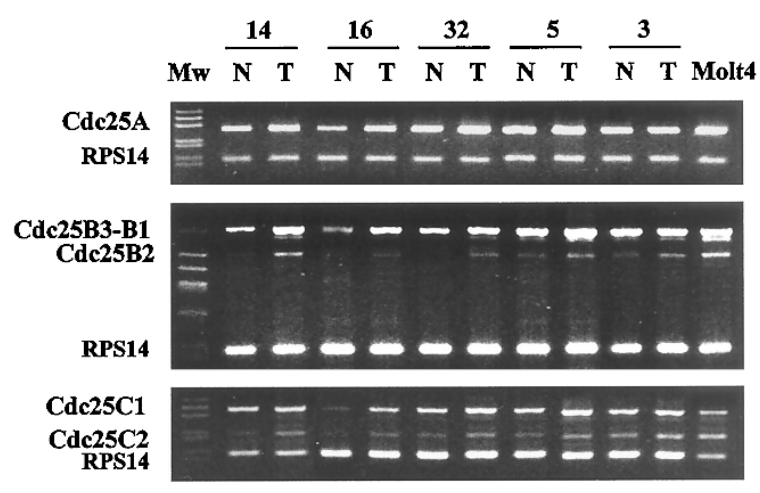

Figure 2.

RT-PCR analysis of cdc25A, B1, B2, B3, B4, C1, and C2 in a series of tumor (T) and normal (N) colon tissues. cdc25A signal was detected in all cases with relatively similar expression levels in normal and tumor samples. cdc25B2 expression was detected in most tumors and in a number of normal tissues. cdc25B1 and B3 splicing isoforms were detected in virtually all cases with relatively higher levels in tumor samples than in normal samples. In addition to the expected cdc25C1 transcript, a new $\mathrm{C} 2$ band was also detected in all tumors and in a number of normal mucosa.

levels in normal and tumor tissues. However, six carcinomas $(40 \%)$ had discordant mRNA and protein expression, all with protein overexpression but similar mRNA levels in normal and tumor samples.

cdc25B. cdc25B mRNA expression was studied by Northern blot in the 34 paired normal and tumor colon tissues. cdc25B expression was higher in tumor samples $(0.7 \pm 1.14$ relative units) than in normal mucosa $(0.22 \pm 0.28$ relative units) $(p<0.005)$. Nineteen tumors (56\%) had a 3-fold increase over the levels found in their corresponding normal mucosa (Fig. 1). No significant correlations were observed between the cdc25B mRNA levels and the clinicopathologic characteristics of the tumors, including site of the tumor, histologic type, infiltration of the bowel wall, or tumor stage. cdc25B mRNA overexpression was present in one of the three patients in Stage I (33\%), and in 10 Stage II patients (50\%) and 8 Stage III patients (73\%). No significant 


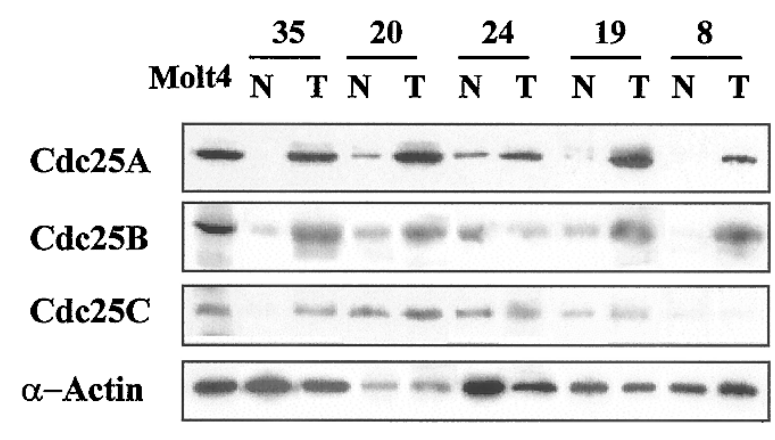

Figure 3.

Western blot analysis of cdc25A, B, and C. cdc25A and B protein overexpression was detected in $53 \%$ and $67 \%$ of tumors, respectively. The levels of $\mathrm{cdc} 25 \mathrm{C}$ protein were lower than those observed for cdc25A and B. Only four tumors $(27 \%)$ overexpressed cdc $25 \mathrm{C}$.

correlations were observed using other cut-off values of the cdc25B mRNA tumor to normal ratio.

To determine the possible differential expression of the cdc25B splicing variants B1, B2, B3, and B4, mRNA from normal and tumor samples was examined by RT-PCR using a pair of primers that selectively amplified all variants (Fig. 2). cdc25B3-B1 splicing isoforms were detected in virtually all cases. cdc25B3 showed a stronger signal with relatively higher levels in tumor than in normal samples. cdc25B2 expression was detected in 27 carcinomas (79\%) but only in 9 of the 34 normal samples $(27 \%)$. In the nine cases in which the cdc25B2 isoform was detected in the normal sample, it was also present in the paired carcinoma. The presence of the cdc25B2 splicing variant was associated with the grade of the differentiation of the tumors $\left(\chi^{2}=3.67, p=0.05\right)$. Thus, cdc25B2 expression was not detected in the well-differentiated adenocarcinomas, but in 21 (84\%) of the moderately differentiated adenocarcinomas and $3(100 \%)$ of the poorly differentiated adenocarcinomas $(p=0.05)$. The three mucinous carcinomas also expressed cdc25B2. cdc25B2 was detected in 2 patients in Stage I (67\%), 14 in Stage II (70\%), and 11 in Stage III (100\%), showing a trend towards correlation of cdc25B2 expression and tumor stage. However, these differences were not statistically significant. The cdc25B4 variant was not detected in any tumor or normal mucosa or in the Molt4 cell line.

To determine whether cdc25B mRNA up-regulation was also associated with protein overexpression, we examined 15 paired normal and cancer colon tissues by Western blot. cdc25B protein expression was detected in all samples with a single band of approximately $67 \mathrm{kd}$. Similar to a previous study in nonHodgkin's lymphomas (Hernández et al, 2000), it was not possible to distinguish the different proteins codified by the alternative-splicing variants of the gene, probably because of the small differences in their molecular weights (Baldin et al, 1997). Ten carcinomas $(67 \%)$ showed higher levels of cdc25B protein expression than their corresponding normal tissues (tumor to normal ratio $>3$-fold) (Fig. 3). Concordant protein and mRNA tumor expression was detected in 11 of 15 tumors $(73 \%)$. Only four tumors showed discordant
mRNA and protein expression, two of them had high protein levels but not high mRNA expression, and two tumors overexpressed cdc25B mRNA but the protein signal was similar between normal and tumor tissues.

The proliferative activity of the tumors was examined by flow cytometry in 29 carcinomas and their respective normal mucosa where suitable material was available. No significant differences were observed in the S-phase fraction in tumors with high cdc25B mRNA overexpression (tumor to normal ratio $>3$ ) (mean value $9 \pm 4 \%$ ), compared with tumors with lower expression levels (mean value $10 \pm 5 \%$ ). The S-phase fraction was also similar in high- and lowexpressing tumors when other cut-off values of cdc25B expression were investigated. Similarly, no differences in the proliferative activity of the tumors were observed regarding the expression of the cdc25B2 isoform. Eighteen of the 29 tumors (62\%) examined were aneuploid. No differences in the expression of cdc25B or cdc25B2 isoform were observed between aneuploid and diploid tumors.

cdc25C. cdc25C mRNA expression was not detected by Northern blot analysis (Fig. 1). To determine whether cdc25C could be expressed at lower levels, we examined these samples by RT-PCR. We observed two different cdc25C transcripts, a larger 338 bp transcript (C1) concordant with the expected size according to the previously described cdc25C cDNA sequence (Gasparotto et al, 1997), and a smaller 214 bp variant (C2) not previously recognized (AF312681) (Fig. 2). The cdc25C1 and $\mathrm{C} 2$ transcripts were detected in all tumors and in $29(85 \%)$ and $19(56 \%)$ of the normal samples, respectively. The cdc $25 \mathrm{C} 1$ expression levels were relatively similar in most normal and tumor samples. However, nine carcinomas (26\%) showed higher levels of expression (tumor to normal ratio $>3$-fold) than the corresponding normal tissues.

cdc25C protein expression was also studied in 15 paired normal and cancer colon tissues by Western blot. cdc25C protein expression was detected in virtually all normal and tumor samples with a single band of approximately $65 \mathrm{kd}$. However, the levels were always lower than those observed for cdc25A and B. Only four tumors overexpressed cdc25C protein. cdc25C 1 mRNA and protein levels were concordant in 12 cases $(80 \%), 3$ of the tumors showed high levels of both protein and mRNA, and 9 cases had similar protein and mRNA expression in the tumor and normal samples.

\section{Characterization of the New cdc25C2 Splicing Isoform and Partial Genomic Organization of cdc25C Gene}

To characterize the new cdc25C2 transcript detected in this study, the amplified cdc25C1 and C2 PCR products were purified and sequenced. The sequence of the cdc25C1 transcript was identical with that previously described (GenBank, M34065). However, the cdc25C2 transcript showed a 124 bp internal deletion concordant with the smaller size of the fragment. Because the genomic organization of the cdc25C gene was not known, it was not possible to determine whether this new transcript was generated 
by an alternative splicing of the normal gene. To investigate this possibility, normal genomic DNA was amplified using two sets of primers flanking the $5^{\prime}$ and $3^{\prime}$ regions of the deleted sequence in the new cdc25C2 transcript (Fig. 4). A fragment of approximately $900 \mathrm{bp}$ was amplified in the $5^{\prime}$ genomic region of the deleted sequence, whereas no amplification was obtained in the $3^{\prime}$ region, probably because of a larger size of the putative intronic sequence. Comparison of the amplified 5' genomic sequence with the GenBank database showed an almost total homology with part of the Homo Sapiens clone DJ0098022 (GenBank, AC004821). This sequence was not previously annotated. The comparative analysis of the genomic sequence included in this clone and the cDNA sequence of the human cdc25C (GenBank, M34065), allowed us to determine the exon-intron organization of the human cdc25C gene from the promoter region to intron 7 (Table 1) (Fig. 4). No further genomic sequences were available in the GenBank database. According to this genomic organization, the cdc25C2 transcript detected in our study lacked the whole exon 4 and 5 sequences (AF312681) (Fig. 4). The smaller size of the intron 3 that was amplified in our normal DNA sample (approximately 900 bp) compared with the genomic sequence in the GenBank database (2429 bp) (Table 1) may be due to a polymorphic difference. The new open reading frame of this transcript generates a stop codon at position 122. Although the antibody used in the Western blot study recognized a domain of the protein encoded by a sequence also present in this cdc25C2 isoform, the putative $15 \mathrm{kd}$ truncated protein derived from this splicing variant was not observed in any case.

\section{cdc25A, B, and C Gene Analysis by Southern Blot}

To determine whether cdc25 mRNA overexpression in colon tumors was associated with amplifications of the genes, genomic DNA from 30 paired cancer and normal colon tissues were examined by Southern blot. No $c d c 25 B$ gene alterations were observed in any tumor, including cases with mRNA overexpression. This indicated that other mechanisms could be implicated in the up-regulation of this gene. In this series of patients, the frequency of the two-allele EcoRI cdc25B polymorphism previously described was similar (A1:

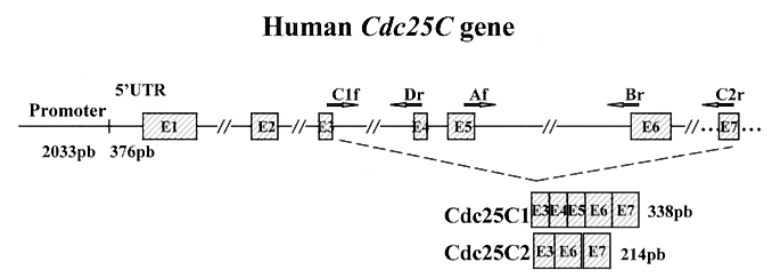

Figure 4.

Schematic representation of the $c d c 25 \mathrm{C}$ intron-exon organization, indicating the relative position of the primers used in this study, and the expected size of the cdc25C1 and C2 cDNA. C1f and C2r were the primers used in the RT-PCR study, whereas $\mathrm{Dr}$, Af, and $\mathrm{Br}$ were the primers used to amplify the $5^{\prime}$ and $3^{\prime}$ genomic regions flanking the deleted sequence in the cdc25C2 variant. Exons 4 and 5 are eliminated in the cdc25C2 splicing variant (AF312681).
0.14 and $A 2: 0.86)$ to that observed in the normal population (A1: 0.13; A2: 0.87) (Hernández et al, 1998). Southern blot analysis of the cdc25A gene showed four bands of $7.9 \mathrm{~kb}, 5.7 \mathrm{~kb}, 2.2 \mathrm{~kb}$, and $1.4 \mathrm{~kb}$, whereas hybridization with the $c d c 25 \mathrm{C}$ probe showed five bands of $7.02 \mathrm{~kb}, 6.6 \mathrm{~kb}, 2.8 \mathrm{~kb}, 2.1 \mathrm{~kb}$, and 0.85 $\mathrm{kb}$. No structural alterations of these genes were detected in any of the tumors including the cases with higher levels of cdc25A and C mRNA in the RT-PCR analysis.

\section{cdc25B Expression and Prognosis}

To evaluate the potential influence of cdc25B mRNA expression levels on the prognosis of the patients, the probabilities of tumor relapse and the overall and cancer-related survivals were analyzed using different cut-offs of the tumor to normal mRNA ratio determined in the Northern blot analysis. When the patients were stratified according to relatively high expression levels (tumor to normal ratio $\geq 4$-fold versus $<4$-fold), a significant association was observed between cdc25B overexpression and the tumor relapse, overall survival, and cancer-related survival of the patients. After a median follow-up period of 54 months, 7 of 11 patients $(64 \%)$ with high levels of cdc25B expression and 6 of 23 patients (26\%) without cdc25B overexpression had tumor relapse $\left(\chi^{2}=4.43, p<0.035\right)$. The probability of tumor relapse at 2 and 5 years was $36 \%$ and $54 \%$, respectively, in patients with cdc25B overexpression, and $17 \%$ and $22 \%$, respectively, in patients without cdc25B overexpression (log-rank = $3.74, p=0.05$ ) (Fig. 5A). At the end of the study, 8 of 11 patients $(73 \%)$ with cdc25B overexpression and 7 of 23 patients $(30 \%)$ without cdc25B overexpression had died $\left(\chi^{2}=3.82, p=0.05\right)$. The probability of overall survival at 2 and 5 years was $64 \%$ and $46 \%$, respectively, in patients with cdc25B overexpression, and $83 \%$ and $69 \%$, respectively, in patients without cdc25B overexpression (log-rank $=4.47, p<0.03$ ). The probability of cancer-related survival at 2 and 5 years was $64 \%$ and $53 \%$, respectively, in patients with cdc25B overexpression, and $86 \%$ and $77 \%$, respectively, in patients without cdc25B overexpression (logrank $=5.16, p<0.02$ ) (Fig. 5B). No correlations between cdc25A and cdc25C expression and survival were observed.

\section{Discussion}

cdc25 genes are a family of dual-specificity phosphatases that activate CDK/cyclin complexes at different points of the cell cycle. Different studies, including experimental and human cancer models, have indicated that $c d c 25 A$ and $B$, but not $C$, may have oncogenic potential, particularly in cooperation with other oncogenic events (Galaktionov et al, 1995, 1996; Spitkovsky et al, 1996). In the present study, we examined the gene structure and expression of cdc25A, B, C, and several splicing variants in a series of 34 paired tumor and normal colorectal tissues. Our results indicated that the cdc25 isoforms are differentially regulated in colorectal 
Table 1. Exon/Intron Junction of the Partial Genomic Sequence of the cdc25C Human Gene ${ }^{a}$

\begin{tabular}{clrrr}
\hline Number & \multicolumn{2}{c}{ Exon/Intron junction } & Exon size & Intron size \\
\hline 1 & & TTGTCTGG gtaaatat & 232 & 1339 \\
2 & ctcaacag AGGAACCC & TGAACTG gtagggag & 95 & 1023 \\
3 & gattgcag GTCACCTG & GCTGGGAT gtaagttc & 46 & 2429 \\
4 & atctgcag GAATCATG & GTAGCCCA gtgagtag & 34 & 116 \\
5 & tttttag GCACAGCT & AAGAAAAT gtgagtgt & 90 & 6440 \\
6 & cttgacag GACAATGG & AAGCAAAG gtaaagtg & 156 & $>729$ \\
\hline
\end{tabular}

${ }^{a}$ Clone DJ0098022 (GenBank, AC004821).

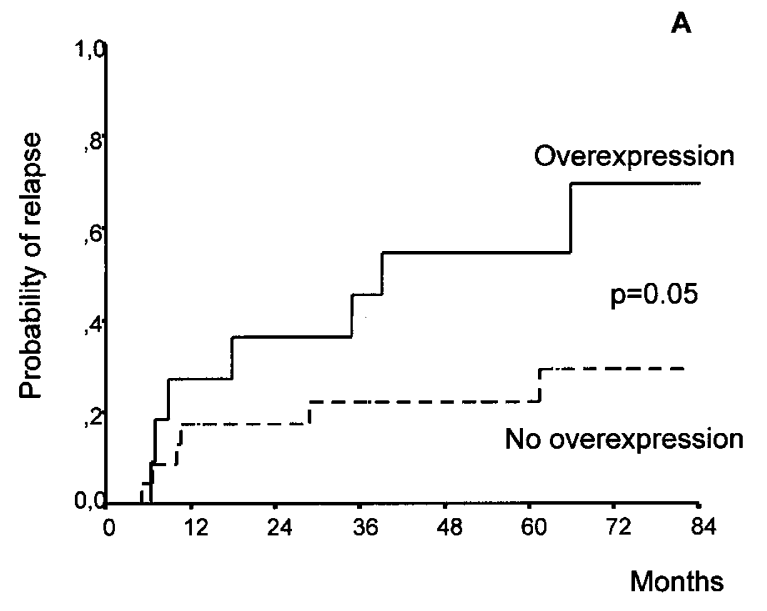

$\mathbf{B}$

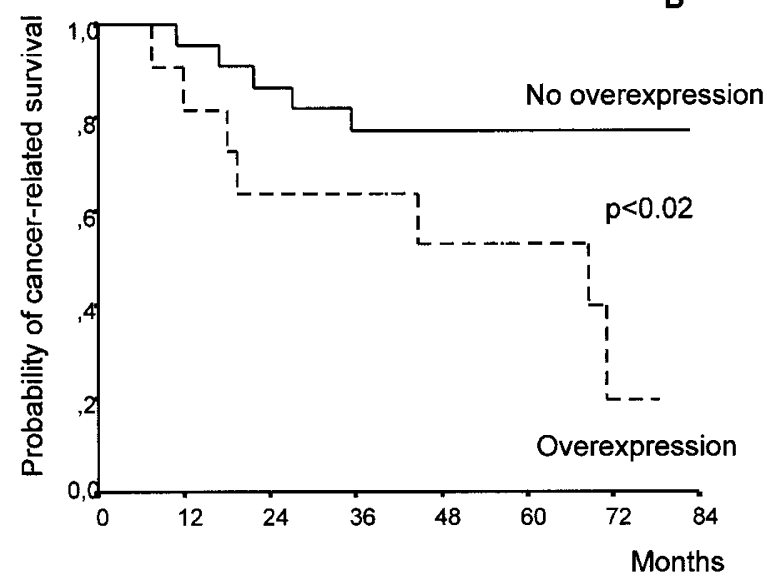

Figure 5.

Probability of tumor relapse $(A)$ and cancer-related survival $(B)$ according to cdc25B overexpression in patients with colorectal cancer.

carcinomas and confirm that cdc25B overexpression may be associated with the progression of these tumors (Takemasa et al, 2000).

In the present study, cdc25B mRNA overexpression was observed in $56 \%$ of the tumors, whereas high cdc25A and C1 mRNA levels were detected in 12\% and $26 \%$ of the carcinomas, respectively. Relatively good correlations between mRNA and protein levels were observed for cdc25B and C. However, cdc25A protein overexpression was observed more frequently than mRNA overexpression, suggesting that posttranscriptional mechanisms may participate in the regulation of cdc25A (Mailand et al, 2000). These results in colorectal carcinomas are similar to previous observations in other tumors, in which cdc25A and B were overexpressed in $35 \%$ to $80 \%$ of cases compared with the respective normal tissues. However, cdc25C overexpression has not been previously observed in other human neoplasms (Gasparotto et al, 1997; Hernández et al, 1998; Kudo et al, 1997; Takemasa et al, 2000; Wu et al, 1998).

The relatively high incidence of cdc25A and $B$ overexpression in different histologic types of tumors (Galaktionov et al, 1995; Gasparotto et al, 1997; Hernández et al, 1998, 2000; Kudo et al, 1997; Ma et al, 1999; Takemasa et al, 2000; Wu et al, 1998; Yao et al, 1999) indicates that cdc25 up-regulation may be a common phenomenon in the development of human malignancies. However, the possible role of these genes in the progression of the tumors is not well known. In a previous study, we observed a significantly higher incidence of cdc25A and B overexpression in aggressive non-Hodgkin's lymphomas than in indolent nonHodgkin's lymphomas (Hernández et al, 1998, 2000). Galaktionov et al (1995) found that cdc25B overexpression in breast carcinomas was associated with poor prognostic parameters, such as a high microvessel density and higher histologic grade. Additionally, patients with cdc25B up-regulation showed shorter disease-free and overall survivals than patients with low cdc25B expression. In a recent study, Takemasa et al (2000) demonstrated that cdc25B protein was overexpressed in $43 \%$ of colorectal carcinomas and that it was an independent prognostic parameter in these patients. In the present study, we analyzed a series of colorectal cancer patients who underwent curative colorectal surgery. cdc25B mRNA up-regulation in these patients was associated with shorter disease-free, overall, and cancer-related survivals. Although the number of cases in the present study is relatively small, these observations confirm, at the mRNA level, the previous findings indicating that cdc25B overexpression may play an important role in the progression of these tumors.

How cdc25B up-regulation participates in tumor progression is not clear. cdc25B is believed to be involved in cell-cycle regulation by activating the cyclin B/cdc2 complexes at the G2/M phase (Izumi and Maller, 1993; Lammer et al, 1998). However, no differences in cell proliferation were detected in our colorectal tumors with high and low cdc25B mRNA levels. Similarly, no correlation was found between cdc25B overexpression and the mitotic index or the Ki-67labeling index in breast and colorectal carcinomas, 
respectively (Galaktionov et al, 1995; Takemasa et al, 2000). Different studies have suggested that cdc25A and $B$ may play roles in the cellular response to DNA damage. Thus, CHK1 kinase may inactivate cdc25A and B phosphatases in response to DNA damage, inducing a cell-cycle arrest that may allow DNA repair (Mailand et al, 2000; Sanchez et al, 1997). However, cdc25B also seems to participate in the control of mitotic spindle formation (Gabrielli et al, 1996). Overexpression of these activating phosphatases in tumors may facilitate the deregulation of these checkpoints, allowing the cell to accumulate progressive DNA alterations. Supporting this hypothesis, cdc25B transgenic animals have an increased susceptibility to develop tumors induced by DNA-damaging agents (Yao et al, 1999).

The mechanisms leading to cdc25 overexpression in human tumors are not well known. cdc25B gene amplification was observed in non-small lung carcinomas using a multiplex PCR technique (Wu et al, 1998). However, these amplifications did not correlate with the levels of mRNA expression. No amplifications or rearrangements of any of the cdc25 genes were observed in our study by Southern blot analysis, implicating other mechanisms in the up-regulation of the $c d c 25$ genes. $c d c 25 A$ and $B$ but not $C$ have been identified as transcriptional targets of $c-m y c$, and c-myc/max binding sites have been found in cdc25A and $B$ regulatory regions (Galaktionov et al, 1996). The c-myc overexpression observed in $60 \%$ to $70 \%$ of colorectal carcinomas (Erisman et al, 1985; Sato et al, 1994; Smith et al, 1993) may play a role in cdc25A and $\mathrm{B}$ overexpression in these tumors.

Different studies have identified up to five different splicing variants of cdc25B gene (B1 to B5) (Baldin et al, 1997; Forrest et al, 1999). These variants are differentially expressed in several cell types and seem to have different mitosis-inducing efficiencies (Baldin et al, 1997). However, their presence in human normal and neoplastic tissues is not well described. Previous studies of cdc25B expression used either Northern blotting or RT-PCR strategies that precluded recognition of these splicing variants. In the present study, we observed that the cdc25B3 isoform is the main splicing variant expressed in normal and tumor colorectal tissues, with relatively higher levels in carcinomas. cdc25B1 was observed at low levels in virtually all normal and tumor samples, although in certain cases it was difficult to distinguish from a strong B3 band. Interestingly, the cdc25B2 isoform was detected in only $27 \%$ of normal tissues but in $79 \%$ of carcinomas. The cdc25B2 variant is more active than B1 and B3 in inducing mitosis in experimental models. Additionally, cdc25B2 is expressed in immortalized fibroblasts but not in diploid primary fibroblasts (Baldin et al, 1997). In a previous study, we showed that the cdc25B2 isoform was not detectable in normal and reactive lymphoid tissues and it was more frequently expressed in aggressive non-Hodgkin's lymphomas than in indolent non-Hodgkin's lymphomas (Hernández et al, 2000). These observations in human tumors are concordant with the higher activity of cdc25B2 in experimental models and suggest that this splicing variant may participate in the pathogenesis of human neoplasms. We have not detected the cdc25B4 variant in any of the tumors nor in the Molt4 cell line, one of the cell lines in which this isoform had been observed previously (Forrest et al, 1999). The reason for this discordance is not clear, but it may be because of the extremely low levels of expression of this variant, as was recognized in the original studies.

Contrary to the relatively high levels of cdc25A and B observed in different tumors, cdc25C does not seem to be up-regulated in malignant neoplasms (Gasparotto et al, 1997; Kudo et al, 1997; Ma et al, 1999; Wu et al, 1998). However, in colorectal tumors we observed cdc25C mRNA and protein overexpression in $26 \%$ and $27 \%$, respectively, of the carcinomas examined. This suggests that the cdc $25 \mathrm{C}$ isoform may also play a role in the pathogenesis of these tumors. In addition to the expected cdc25C isoform, we detected a new cdc25C2 splicing variant, which was more frequently found in tumors (100\%) than in normal mucosa (56\%). This variant was not observed in a previous cdc25C study in head and neck carcinomas using the same set of primers (Gasparotto et al, 1997), suggesting that this splicing isoform may have a tissue specificity. The molecular characterization of this variant and its $5^{\prime}$ genomicflanking region allowed us to annotate the cdc25C genomic sequence deposited in GenBank, and to identify the exon-intron structure of the gene. This new cdc25C2 variant lacked exons 4 and 5 and coded for a truncated protein without the catalytic domain. This particular structure and the lack of detection of the predicted shorter protein in the Western blot analysis question the possible functional role of this splicing isoform in the pathogenesis of the tumors.

In conclusion, these findings indicate that cdc25 isoforms and splicing variants are differentially regulated in colorectal carcinomas and may participate in the development of these tumors. Additionally, the correlation between cdc25B mRNA levels and the survival of the patients also suggests that this particular isoform may be involved in the progression of the disease.

\section{Material and Methods}

\section{Patients and Tissues}

Thirty-four colorectal carcinomas were randomly collected from patients who underwent curative colorectal surgery in our hospital between 1993 and 1994. The patients comprised 18 women and 16 men, with a median age of 67 years (range: 32 to 91 years). All patients had fully documented clinical histories and staging investigations. The median follow-up period was 54 months (range: 8 to 82 months). Samples from tumor areas and matched normal mucosa were snapfrozen in isopentane precooled in liquid nitrogen and stored at $-80^{\circ} \mathrm{C}$ until studied. The normal mucosa samples were obtained at least $10 \mathrm{~cm}$ away from the tumor and dissected from the underlying muscle layer. The remaining specimen was fixed in formalin and routinely processed. The carcinomas included welldifferentiated $(n=2,6 \%)$, moderately differentiated $(n$ 
$=26,76 \%)$, poorly differentiated ( $n=3,9 \%$ ), and mucinous carcinomas ( $n=3,9 \%$ ). The distribution of these tumors according to the TNM system was: Stage I, 3 cases (9\%); Stage II, 20 cases (59\%); and Stage III, 11 cases (32\%). The cancer cell line Molt-4 was used as a control in this study and was obtained from the American Type Culture Collection (Rockville, Maryland. Cells were grown in RPMI (Life Technologies, Inc., Paisley, United Kingdom) containing 15\% FCS in a $5 \% \mathrm{CO}_{2}$-humidified atmosphere.

\section{RNA Extraction and Northern Blot Analysis}

Total RNA was isolated from frozen tissues from 34 colorectal carcinomas and correspondent normal mucosa by guanidine isothiocyanate extraction and cesium chloride gradient centrifugation, as described previously (Glissin et al, 1974). Cryostat sections of these cases were previously examined to determine the proportion of normal and neoplastic tissue present in each sample. Tumor samples were selected only if more than $75 \%$ of the section was carcinoma. Fifteen micrograms of total RNA were subjected to electrophoresis on a denaturing $1.2 \%$ agarose formaldehyde gel and transferred to Hybond-N membranes (Amersham International, Buckinghamshire, United Kingdom). The membranes were prehybridized, hybridized with the $\operatorname{cdc} 25 A, B$, and $C$ probes, and washed, as described previously (Hernández et al, 1998). Probes were radiolabeled using a random primer DNAlabeling kit (Promega Corporation, Madison, Wisconsin) with $\left(\alpha^{-}{ }^{32} \mathrm{P}\right) \mathrm{dCTP}$. Intensities of cdc25 mRNA transcripts were normalized to the 28S RNA band. The signals were quantified with the Quantity-One Software (version 4.0.1, BioRad, Hercules, California). In each blot, total RNA from the Molt- 4 cell line was also included as a positive control. Hybridization values of this cell line were used to normalize the signal between blots.

\section{cDNA Synthesis and PCR Analysis}

The reverse transcription reaction was performed using the SuperScript preamplification kit (GIBCO BRL, Gaithersburg, Maryland). One microgram of total RNA was subjected to reverse transcription with random hexamers and 200 units of SuperScript II RT (GIBCO $\mathrm{BRL})$. After the retrotranscriptase reverse reaction, 2 units of RNAase $\mathrm{H}$ was always added to remove the RNA template from the cDNA. PCR reactions were performed in a total volume of $50 \mu \mathrm{l}$, using $1 \mu \mathrm{l}$ of cDNA. The primers used for cdc25A (273 bp) amplification were A1 forward (5'GAG GAG TCT CCA CCT GGA AGT ACA) and A2 reverse (5' GCC ATT CAA AAC AGA TGC CAT AA) (Gasparotto et al, 1997); for cdc25B4 (627 bp), B3 (522 bp), B1 (480 bp), and B2 (399 bp) amplification were B1 forward (5'GCT TCC TCG CCG GTC ACC AC) and B2 reverse (5'CCT GCG GCT GGC CCA CTC) (Baldin et al, 1997); and for cdc25C1 (338 bp) and C2 (214 bp) amplification were C1 forward (5' CAG GAA GTG CAT TTA GCT GGG ATG) and C2 reverse (5' ATC GAC GGG GAG CGA
TAT AGG C) (Gasparotto et al, 1997). In each reaction, the RPS14 ribosomal mRNA (143 bp) was coamplified as a control using the primers $\mathrm{S} 14$ forward (5' GGC AGA CCG AGA TGA ATC CTC A) and S14 reverse (5' CAG GTC CAG GGG TCT TGG TCC) (Foley et al, 1993). The amplification conditions were $94^{\circ} \mathrm{C}$ for 1 minute, $56^{\circ} \mathrm{C}$ (for cdc25A), $63^{\circ} \mathrm{C}$ (for cdc25B4, $\mathrm{B} 3, \mathrm{~B} 1$, and $\mathrm{B} 2$ ) or $60^{\circ} \mathrm{C}$ (for cdc25C) for 45 seconds and $72^{\circ} \mathrm{C}$ for 1 minute, during 30 cycles, with a common additional elongation step of $72^{\circ} \mathrm{C}$ for 10 minutes. All of these reactions were carried out in a PE480 thermocycler (Perkin-Elmer Cetus, Norwalk, Connecticut). The amplified products were separated by electrophoresis in a $2 \%$ agarose Tris-borate EDTA gel, and the bands were visualized by ethidium bromide staining. RNA extracted from the Molt4 cell line was used as a positive control.

\section{Western Blotting}

Protein extraction was obtained from additional frozen tissue available in 15 carcinomas and their respective normal mucosa as previously described (Jares et al, 1996). Protein extract from the Molt4 cell line was used as a positive control. Thirty micrograms of total cellular protein were run per lane on a $10 \%$ SDSpolyacrylamide gel and electroblotted to a nitrocellulose membrane (Amersham Corporation, Arlington Heights, Illinois). The membranes were blocked by overnight incubation in $5 \%$ dry milk and $0.1 \%$ Tween-20 at $4^{\circ} \mathrm{C}$. The blocked membranes were incubated with polyclonal antibodies to cdc25A (Santa Cruz Biochemicals, Santa Cruz, California), and cdc25B (Pharmingen, San Diego, California), and a monoclonal antibody to cdc25C (Pharmingen) for 1.5 hours, washed with $0.1 \%$ Tween-20 in PBS, and incubated with a sheep anti-rabbit or anti-mouse secondary antibody conjugated to horseradish peroxidase (Amersham Corporation). A monoclonal antibody to $\alpha$-actin was used as a loading control in all cases. After washing, antibody binding was detected by chemoluminescence detection procedures according to the manufacturer's recommendations (ECL, Amersham Corporation). Intensities of the cdc25 proteins were normalized to the actin band. The signals were quantified with the Quantity-One Software (version 4.0.1, BioRad). In each blot, total protein from Molt-4 cell line was also included as a positive control.

\section{Sequencing Analysis}

To confirm the specificity of the RT-PCR amplification signals, PCR products were purified and sequenced in selected cases. The amplified products were subjected to electrophoresis in a $2 \%$ agarose Tris-borate EDTA gel and the bands were excised and purified by an Agarose Gel Extraction Protocol (QIAEX II, Qiagen, Hilden, Germany). The DNA extracted was sequenced with a Taq DyeDeoxy dRhodamine Terminator Cycle Sequencing method using an ABI PRISM automated DNA sequencer (Applied Biosystems, Foster City, California). Sequences were compared with the Gen- 
Bank sequence database (GenBank: M34065, AF036233, and M81933).

\section{DNA Extraction and Southern Blot Analysis}

Genomic DNA was obtained from 30 paired normal and cancer colon frozen tissues, using Proteinase K/RNAase treatment and phenol-chloroform extraction. Fifteen micrograms were digested with EcoRI, separated on $0.8 \%$ agarose gels, and transferred to Hybond-N membranes (Amersham International). The membranes were prehybridized, hybridized with the $c d c 25 A, c d c 25 B$, and $c d c 25 C$ probes, as previously described (Hernández et al, 1998), and subsequently hybridized with the $\beta$-actin probe as a loading control. The signals of the Southern blot analysis were quantified with the same system used in the Northern blot analysis.

\section{Flow Cytometry}

Proliferative parameters and DNA ploidy of the tumors were studied by flow cytometry in 29 paired normal and cancer colon samples in which suitable material was available. The analysis was performed on 50 -mm-thick sections obtained from formalin-fixed, paraffinembedded tissues using a technique previously described (Hedley et al, 1983). The nuclear samples were stained with propidium iodide and analyzed with an Epics Profile II flow cytometer (Coulter Company, Hialeah, Florida). Non-neoplastic cells in the section under study were used as the internal standard of the diploid channel as recommended by the DNA Cytometry Consensus Conference. Discrimination of doublets and aggregates was performed on the basis of the pulse peak versus pulse area analysis with the Power Pack system (Coulter Company). Single-parameter histograms analyzed with the Multicycle software (Phoenix Flow Systems, San Diego, California) were classified according to the guidelines on nomenclature of the Society for Analytical Cytology. The proliferative fraction (S-phase) of the tumor and normal tissues was analyzed using the Power Pack doublet discrimination system (Coulter Company).

\section{Statistical Analysis}

Continuous variables were expressed as mean \pm standard deviation. Differences in the relative frequency of cdc25 overexpression and correlation between qualitative variables were evaluated by means of the $\chi^{2}$ test. Continuous variables were compared by means of the Student $t$ test or Mann-Whitney $U$ test depending on their distribution. Correlation between continuous variables was assessed by regression analysis. Probability of tumor relapse, overall survival, and cancer-related survival was calculated according to the Kaplan-Meier method. Comparison between curves was made by the log-rank test. Starting time for survival analysis was the day of surgical resection of primary colorectal cancer. All calculations were performed with the SPSS software package (SPSS Inc., Chicago, Illinois).

\section{References}

Baldin V, Cans C, Superti-Furga G, and Ducommun B (1997). Alternative splicing of the human CDC25B tyrosine phosphatase. Possible implications for growth control? Oncogene 14:2485-2495.

el-Deiry WS, Tokino T, Waldman T, Oliner JD, Velculescu VE, Burrell M, Hill DE, Healy E, Rees JL, and Hamilton SR (1995). Topological control of p21WAF1/CIP1 expression in normal and neoplastic tissues. Cancer Res 55:2910-2919.

Erisman MD, Rothberg PG, Diehl RE, Morse CC, Spandorfer JM, and Astrin SM (1985). Deregulation of c-myc gene expression in human colon carcinomas is not accompanied by amplification or rearrangement of the gene. Mol Cell Biol 5:1969-1976.

Esteller M, Tortola S, Toyota M, Capella G, Peinado MA, Baylin SB, and Herman JG (2000). Hypermethylationassociated inactivation of p14(ARF) is independent of p16(INK4a) methylation and p53 mutational status. Cancer Res 60:129-133.

Foley KP, Leonard MW, and Engel JD (1993). Quantitation of RNA using the polymerase chain reaction. Trends Genet 9:380-384.

Forrest A, McCormack A, DeSouza C, Sinnamon J, Tonks I, Hayward N, Ellem K, and Gabrielli B (1999). Multiple splicing variants of cdc25B regulate $\mathrm{G} 2 / \mathrm{M}$ progression. Biochem Biophys Res Commun 260:510-515.

Gabrielli B, DeSouza P, Tonks I, Clark J, Hayward N, and Ellem K (1996). Cytoplasmic accumulation of cdc25B phosphatase in mitosis triggers centrosomal microtubule nucleation in HeLa cells. J Cell Sci 109:1081-1093.

Galaktionov K and Beach D (1991). Specific activation of cdc25 tyrosine phosphatases by B-type cyclins: Evidence for multiple roles of mitotic cyclins. Cell 67:1181-1194.

Galaktionov K, Chen X, and Beach D (1996). Cdc25 cell-cycle phosphatase as a target of c-myc. Nature 382:511-517.

Galaktionov K, Lee AK, Eckstein J, Draetta G, Meckler J, Loda M, and Beach D (1995). CDC25 phosphatases as potential human oncogenes. Science 269:1575-1577.

Gasparotto D, Maestro R, Piccinin S, Vulkosavljevic T, Barzan L, Sulfaro S, and Boiocchi M (1997). Overexpression of CDC25A and CDC25B in head and neck cancers. Cancer Res 57:2366-2368.

Girlando S, Slomp P, Caffo O, Amichetti M, Togni R, Dvornik G, Tomio L, Galligioni E, Dalla Palma P, and Barbareschi M (1999). p21 expression in colorectal carcinomas: A study on 103 cases with analysis of p53 gene mutation/expression and clinic-pathological correlations. Virchows Arch 435:559565.

Glissin V, Crkvenjakov R, and Byus C (1974). Ribonucleic acid isolated by cesium chloride centrifugation. Biochemistry 13:2633-2637.

Hedley DW, Friedlander HL, Taylor JW, Rugg CA, and Musgrove EA (1983). Method for analysis of cellular DNA content of paraffin-embedded pathological material using flow-cytometry. J Histochem Cytochem 31:1333-1335.

Herman JG, Merlo A, Mao L, Lapidus RG, Issa JP, Davidson NE, Sidransky D, and Baylin SB (1995). Inactivation of the CDKN2/p16/MTS1 gene is frequently associated with aberrant DNA methylation in all common human cancers. Cancer Res 55:4525-4530. 
Hernández $S$, Hernández L, Beà $S$, Cazorla $M$, Fernández $P$, Nadal A, Muntané J, Mallofré C, Montserrat E, Cardesa A, and Campo E (1998). cdc25 cell cycle activating phosphatases and c-myc expression in human non-Hodgkin's lymphomas. Cancer Res 58:1762-1767.

Hernández $S$, Hernández L, Beà $S$, Pinyol $M$, Nayach I, Bellosillo B, Nadal A, Ferrer A, Fernández P, Montserrat E, Cardesa A, and Campo E (2000). cdc25A and the splicing variant cdc25B2, but not cdc25B1, -B3 or -C are overexpressed in aggressive human non-Hodgkin's lymphomas. Int J Cancer 89:148-152.

Hoffmann I, Clarke PR, Marcote MJ, Karsenti E, and Draetta $G$ (1993). Phosphorylation and activation of human cdc25C by cdc2-cyclin $B$ and its involvement in the self-amplification of MPF at mitosis. EMBO J 12:53-63.

Hoffmann I, Draetta G, and Karsenti E (1994). Activation of the phosphatase activity of human cdc25A by a cdk2-cyclin E dependent phosphorylation at the G1/S transition. EMBO $J$ 13:4302-4310.

Hunter T and Pines J (1994). Cyclins and cancer. II: Cyclin D and CDK inhibitors come of age. Cell 79:573-582.

Igarashi $M$, Nagata A, Jinno S, Suto K, and Okayama $H$ (1991). Wee1(+)-like gene in human cells. Nature 353:80-83.

Izumi T and Maller JL (1993). Elimination of cdc2 phosphorylation sites in the cdc25 phosphatase blocks initiation of M-phase. Mol Biol Cell 4:1337-1350.

Jares P, Campo E, Pinyol M, Bosch F, Miquel R, Fernandez PL, Sanchez-Beato M, Soler F, Perez-Losada A, Nayach I, Mallofre C, Piris MA, Montserrat E, and Cardesa A (1996). Expression of retinoblastoma gene product $(\mathrm{pRb})$ in mantle cell lymphomas. Correlation with cyclin D1 (PRAD1/CCND1) mRNA levels and proliferative activity. Am J Pathol 148: 1591-1600.

Jinno S, Suto K, Nagata A, Igarashi M, Kanaoka Y, Nojima H, and Okayama H (1994). Cdc25A is a novel phosphatase functioning early in the cell cycle. EMBO J 13:1549-1556.

Kudo Y, Yasui W, Ue T, Yamamoto S, Yokozaki H, Nikai H, and Tahara E (1997). Overexpression of cyclin-dependent kinase-activating CDC25B phosphatase in human gastric carcinomas. Jpn J Cancer Res 88:947-952.

Lammer C, Wagerer S, Saffrich R, Mertens D, Ansorge W, and Hoffmann I (1998). The cdc25B phosphatase is essential for G2/M phase transition in human cells. J Cell Sci 111: 2445-2453.

Ma Z, Chua S, DeMayo F, and Tsai S (1999). Induction of mammary gland hyperplasia in transgenic mice overexpressing human Cdc25B. Oncogene 18:4564-4576.

Mailand N, Falck J, Lukas C, Syljuasen R, Welcker M, Bartek $\mathrm{J}$, and Lukas $\mathrm{J}$ (2000). Rapid destruction of human Cdc25A in response to DNA damage. Science 288:1425-1429.

Nagata A, Igarashi M, Jinno S, Suto K, and Okayama $H$ (1991). An additional homolog of the fission yeast cdc25+ gene occurs in humans and is highly expressed in some cancer cells. New Biol 3:959-968.

Norbury C, Blow J, and Nurse P (1991). Regulatory phosphorylation of the $\mathrm{p} 34 \mathrm{cdc} 2$ protein kinase in vertebrates. EMBO J 10:3321-3329.
Risio M, Coverlizza S, Ferrari A, Candelaresi GL, and Rossini FP (1988). Immunohistochemical study of epithelial cell proliferation in hyperplastic polyps, adenomas and carcinomas of the large bowel. Gastroenterology 94:899-906.

Sadhu K, Reed SI, Richardson H, and Russell P (1990). Human homolog of fission yeast cdc25 mitotic inducer is predominantly expressed in G2. Proc Natl Acad Sci USA 87:5139-5143.

Sanchez Y, Wong C, Thoma R, Richman R, Wu Z, PiwnicaWorms H, and Elledge S (1997). Conservation of the Chk1 checkpoint pathway in mammals: Linkage of DNA damage to CDK regulation through Cdc25. Science 277:1497-1501.

Sato K, Mayahara M, Saito T, and Kobayashi M (1994). c-myc mRNA overexpression is associated with lymph node metastasis in colorectal cancer. Eur J Cancer 30A:1113-1117.

Sgambato A, Ratto C, Faraglia B, Merico M, Ardito R, Schinzari G, Romano G, and Cittadini AR (1999). Reduced expression and altered subcellular localization of the cyclindependent kinase inhibitor p27(KIP1) in human colon cancer. Mol Carcinog 26:172-179.

Sherr C (1996). Cancer cell cycles. Science 274:1672-1677.

Sherr CJ and Roberts JM (1995). Inhibitors of mammalian G1 cyclin-dependent kinases. Genes Dev 9:1149-1163.

Smith D, Myint T, and Goh H (1993). Over-expression of the c-myc proto-oncogene in colorectal carcinomas. $\mathrm{Br} \mathrm{J}$ Cancer 68:407-413.

Spitkovsky D, Jansen-Durr P, Karsenti E, and Hoffman I (1996). S-phase induction by adenovirus E1A requires activation of cdc25A tyrosine phosphatase. Oncogene 12:2549-2554.

Strausfeld U, Fernandez A, Capony JP, Girard F, Lautredou N, Derancourt J, Labbe JC, and Lamb NJ (1994). Activation of p34cdc2 protein kinase by microinjection of human cdc25C into mammalian cells. Requirement for prior phosphorylation of cdc25C by p34cdc2 on sites phosphorylated at mitosis. J Biol Chem 269:5989-6000.

Takemasa I, Yamamoto H, Sckimoto M, Ohue M, Noura S, Miyake Y, Matsumoto T, Aihara T, Tomita N, Tamaki Y, Sakita I, Kikkawa N, Matsuura N, Shiozaki H, and Monden M (2000). Overexpression of CDC25B phosphatase as a novel marker of poor prognosis of human colorectal carcinoma. Cancer Res 60:3043-3050.

Viale G, Pellegrini C, Mazzarol G, Maisonneuve P, Silverman $\mathrm{ML}$, and Bosari S (1999). p21WAF1/CIP1 expression in colorectal carcinoma correlates with advanced disease stage and p53 mutations. J Pathol 187:302-307.

Wu W, Fan Y, Kemp B, Walsh G, and Mao L (1998). Overexpression of cdc25A and cdc25B is frequent in primary non-small cell lung cancer but is not associated with overexpression of c-myc. Cancer Res 58:4082-4085.

Yamamoto H, Monden T, Miyoshi H, Izawa H, Ikeda k, Tsujie M, Ohnishi T, Sekimoto M, Tomita N, and Monden M (1998). Cdk2/ cdc2 expression in colon carcinogenesis and effects of cdk2/cdc2 inhibitor in colon cancer cells. Int J Oncol 13:233-239.

Yao Y, Slosberg E, Wang L, Hibshoosh H, Zhang Y, Xing W, Santella R, and Weinstein B (1999). Increased susceptibility to carcinogen-induced mammary tumors in MMTV-Cdc25B transgenic mice. Oncogene 18:5159-5166. 